\title{
Nasopharyngeal Papillary Adenocarcinoma
}

National Cancer Institute

\section{Source}

National Cancer Institute. Nasopharyngeal Papillary Adenocarcinoma. NCI Thesaurus. Code C54400.

A rare, low-grade exophytic adenocarcinoma with papillary growth that arises from the epithelium of the nasopharynx. If it is completely removed, the prognosis is excellent. 